\title{
Alimentation d'une pompe immergée à partir d'un générateur photovoltaique à l'aide d'un onduleur triphasé à transistor
}

\author{
M. Barlaud, G. Sow et Ch. Masselot \\ Laboratoire d'Energie Solaire de l'Ecole Nationale Supérieure Universitaire de Technologie de Dakar (E.N.S.U.T.) : \\ Groupe d'Electronique et d'Automatique Appliquée, I.U.T., BP 5085, Dakar-Fann, Sénégal
}

(Reçu le 10 juillet 1979, révisé le 5 novembre 1979, accepté le 8 novembre 1979)

\begin{abstract}
Résumé. - La station de pompage alimentée par un générateur photovoltaïque que nous avons réalisée est constituée par un ensemble électronique hacheur-onduleur triphasé et un groupe moteur asynchrone-pompe centrifuge immergé. Le hacheur dévolteur asservi est un convertisseur continu-continu qui impose au générateur un fonctionnement à puissance maximale quelles que soient les conditions météorologiques.

Il alimente un onduleur triphasé à fréquence variable qui transmet par liaison électrique au moteur toute la puissance disponible aux bornes du générateur photovoltaïque avec un excellent rendement. Les protections actives contre les surintensités protègent également le moteur et confèrent à l'ensemble une excellente sécurité de fonctionnement. Enfin, la station de pompage est entièrement automatique. Elle satisfait aux exigences d'économie, d'autonomie et de fiabilité qui sont nécessaires à son implantation sur un site isolé du Sahel.
\end{abstract}

\begin{abstract}
The photovoltaic generator powered pumping station developed by us consists of a three-phase electronic chopper-inverter unit and an asynchronous motor/underwater centrifugal pump set. The servo chopper step-down transformer is a dc to dc converter which impels the generator to operate at peak power irrespective of weather conditions.

It feeds a variable frequency three-phase inverter which, by way of an electric linkage, transmits total power available at the terminals of the photovoltaic generator to the motor, at high efficiency. The active protecting devices against excess current also protect the motor, thus rendering the whole assembly extremely reliable. Moreover, the pumping station is fully automatic. It meets the three criteria - economy, independence and reliability - necessary for use on a suitable site in the Sahel.
\end{abstract}

1. Introduction. - Malgré les efforts déployés par de nombreux laboratoires pour réduire le prix de revient des cellules photovoltaïques, les panneaux de photopiles proposés par les constructeurs restent encore chers ( $10 \$$ le Watt crête) et leur utilisation ne peut être envisagée que dans les régions éloignées des centres industriels. Ceci est le cas en particulier pour l'exhaure de l'eau nécessaire à la vie des hommes et des animaux qui vivent dans les milieux ruraux isolés du Sahel.

Dans ces conditions, l'utilisation d'un générateur photovoltaïque est soumise aux impératifs suivants : misé

- Le rendement des installations doit être opti-

- La fiabilité des ensembles générateur-récepteur doit être excellente.

- L'intervention de l'homme doit être la plus rare possible. C'est dans cet esprit que nous avons fait évoluer la station de pompage expérimentale de l'IUT de Dakar.
Afin d'éviter l'utilisation d'accumulateurs coûteux et de rendement médiocre [1], le couplage du générateur photovoltaïque au moteur asynchrone (Fig. 1) qui alimente la pompe centrifuge immergée est réalisé directement au moyen d'un ensemble hacheuronduleur triphasé à transistor et l'énergie est stockée sous forme d'eau montée dans un réservoir. Le hacheur placé en tête $[2,3]$ oblige le générateur photovoltaique à fonctionner à puissance maximale quel que soit l'éclairement. La technologie à transistor a été choisie à cause des faibles pertes en commutation et en conduction. Elle autorise en outre une fréquence de hachage élevée, ce qui réduit la dimension des cellules de filtrage.

Pour conserver un couple maximum sur l'arbre $\mathrm{du}$ moteur, l'onduleur doit fonctionner à puissance variable proportionnelle à la tension d'alimentation du moteur asynchrone.

On dispose en surface d'une source d'énergie triphasée qui peut être transmise par câble électrique 


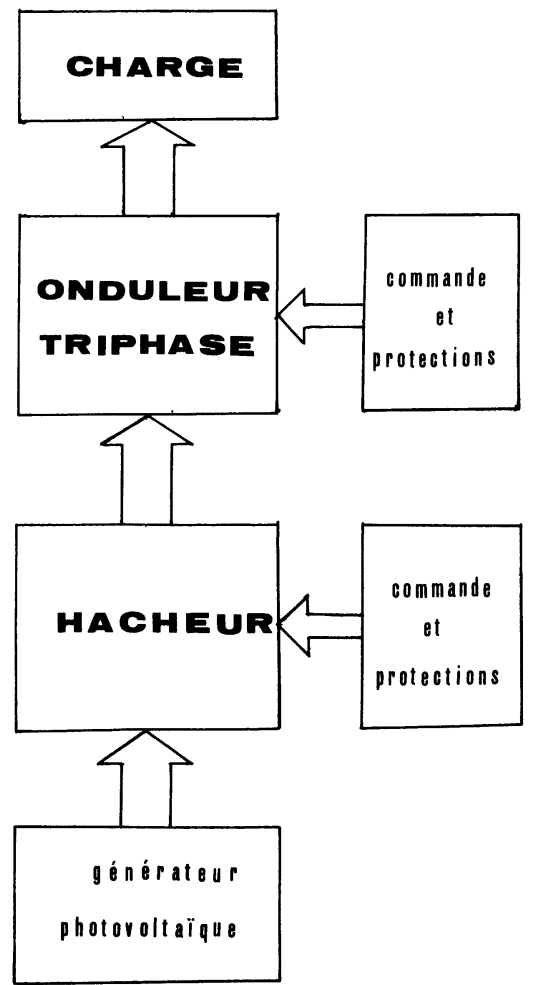

Fig. 1. - Schéma synoptique de la station de pompage.

[Synoptic diagram of the pumping station.]

au fond du puits au groupe moto-pompe immergé, ce qui évite l'utilisation d'un moteur continu en surface associé à une transmission mécanique de grande longueur source de pertes importantes.

Ce dispositif qui transmet au moteur avec un excellent rendement $\left(\eta_{\operatorname{MAX}} \simeq 95 \%\right)$ toute la puissance disponible aux bornes du générateur photovoltaïque permet l'exhaure de l'eau des nappes phréatiques profondes. Les protections actives contre les surintensités conçues au niveau des semi-conducteurs protègent également le moteur et confèrent à l'ensemble une excellente fiabilité. Enfin, la station de pompage est entièrement automatique et ne demande aucune intervention en dehors de l'entretien inhérent aux groupes immergés.

2. Adaptation. - Une installation photovoltaïque comprend un générateur réalisé à l'aide de panneaux de photopiles et un ensemble récepteur. Adapter l'installation consiste à transférer à la charge la puissance maximale disponible aux bornes du générateur.

L'hyperbole $V I=$ Cte tangente à la caractéristique $I=f(v)$ d'un générateur photovoltaïque (Fig. 2) soumis à un éclairement donné définit le point de fonctionnement à puissance maximum du générateur pour cet éclairement. Il est remarquable de constater que ce point de fonctionnement à puissance maximale se déplace sur une droite à tension constante $V \simeq V_{\text {opt }}$ lorsque l'éclairement varie. Or l'énergie solaire reçue

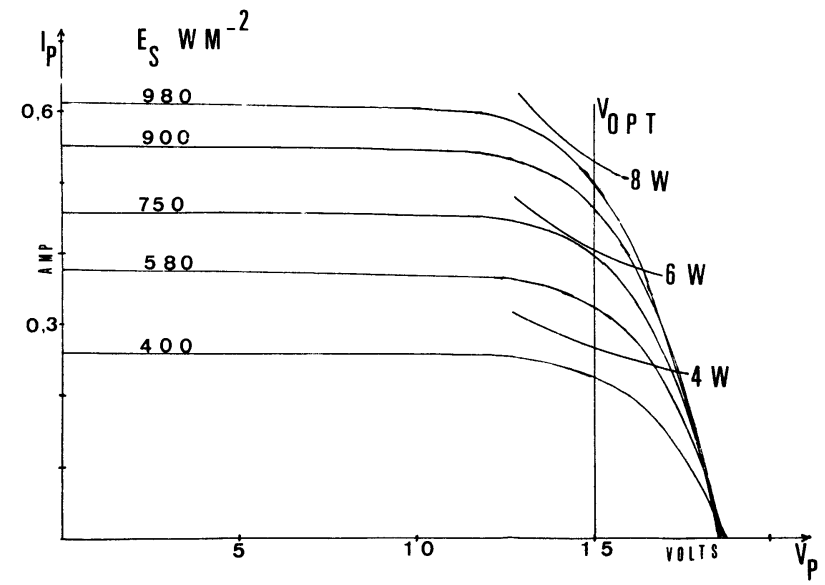

Fig. 2. - Caractéristiques courant-tension d'un panneau de photopiles RTC BPX 147 A pour divers ensoleillements.

[Current versus voltage characteristics of a photocell panel RTC BPX $147 \mathrm{~A}$ at different solar intensities.]

en un lieu donné dépend de l'heure et des conditions météorologiques si bien que la charge du générateur photovoltaique doit être du type force contre électromotrice pure si l'on souhaite fonctionner à puissance maximum quel que soit l'éclairement. Malheureusement, un ensemble onduleur triphasé-machine asynchrone-pompe centrifuge alimenté à puissance variable ne présente pas la caractéristique couranttension souhaitée.

Nous avons réalisé le fonctionnement à puissance maximum en utilisant un adaptateur électronique idéal constitué par un convertisseur continu-continu asservi qui permet de transférer la puissance maximale disponible aux bornes du générateur photovoltaïque à n'importe quelle charge quel que soit l'éclairement.

Ce convertisseur est un interrupteur commandé qui réalise une transformation de l'énergie continue avec un rapport de transformation variable. En effet si l'on considère un hacheur dévolteur (Fig. 3) de période $T$ et de durée de conduction $\theta$, la tension moyenne appliquée à la charge est :

$$
V_{2}=E \frac{\theta}{T}=R E
$$

où $R$ est le rapport cyclique.

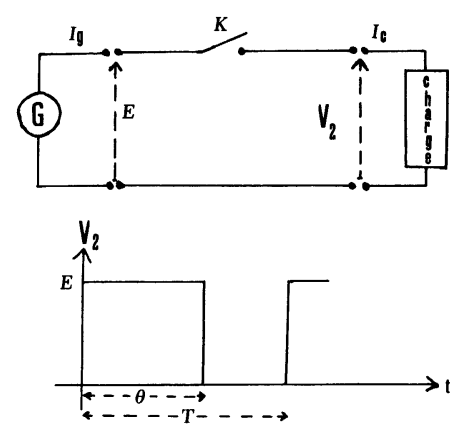

Fig. 3. - Principe d'un hacheur convertisseur continu-continu.

[Principle of a dc to dc chopper adaptor.] 
Un interrupteur commandé peut être un thyristor ou un transistor, nous avons choisi la technologie à transistor de puissance qui permet d'obtenir d'excellents rendements, et autorise une fréquence de commutation élevée, ce qui conduit à une réduction des cellules de filtrage.

Le convertisseur utilisé en pratique (Fig. 4) comprend un transistor de puissance $T_{p}$ fonctionnant en interrupteur, deux filtres $\mathrm{C}$ et $\mathrm{L}$, et un système électronique d'asservissement. Les filtres ont pour rôle d'assurer un débit continu du générateur et une alimentation continue de l'onduleur. La diode de roue libre $\mathrm{D}_{\mathrm{RL}}$ permet la circulation du courant dans la charge lorsque le transistor est bloqué et la diode $\mathrm{D}^{\prime}$ protège le transistor contre les tensions inverses.

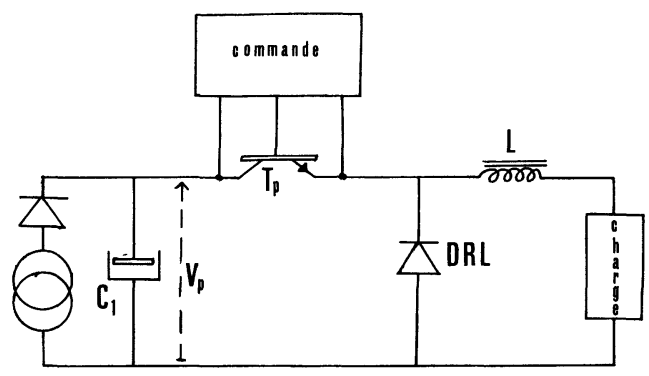

Fig. 4. - Schéma de principe du hacheur adaptateur de puissance. [Skeleton diagram of the power chopper adaptor.]

Le circuit de commande et de protection (Fig. 5) comprend une base de temps, un amplificateur comparateur $\mathrm{C}_{1}$, un comparateur à hystérésis $\mathrm{C}_{2}$, un circuit de mise en forme des impulsions, un circuit d'amplification des impulsions et un bistable de commande de la base du transistor de puissance. Le comparateur $\mathrm{C}_{1}$ élabore une tension d'erreur entre une fraction de la tension des photopiles et une tension de référence correspondant au fonctionnement optimal.

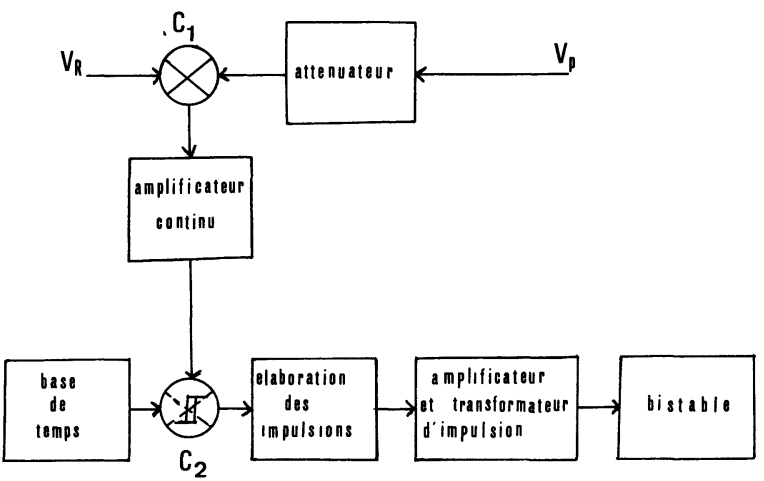

Fig. 5. - Schéma synoptique de l'asservissement au point de fonctionnement optimal du générateur photovoltaïque.

[Synoptic diagram of the servo mechanism at optimum operating point of the photovoltaic generator.]
Cette tension de référence modifie le rapport cyclique des impulsions délivrées par le comparateur à hystérésis $\mathrm{C}_{2}$ et la base de temps de manière à maintenir la tension des photopiles au voisinage de sa valeur optimale. Ces impulsions convenablement mises en forme sont ensuite amplifiées et transmises au bistable de commande du transistor de puissance par l'intermédiaire de transformateurs d'impulsion qui ont également un rôle de transformateur d'isolement.

3. Commande et protections du transistor de puissance. - Le choix du dispositif de commande conditionne la fiabilité de l'électronique et du groupe motopompe.

3.1 Commande DE LA SATURATION DU TRANSISTOR DE PUISSANCE. - Les impulsions de commande sont transmises à un montage bistable (Fig. 6) qui assure la saturation (conduction) et le blocage (ouverture) du transistor de puissance $T_{p}$, ainsi que la détection de toute surintensité qui entraînerait sa désaturation.
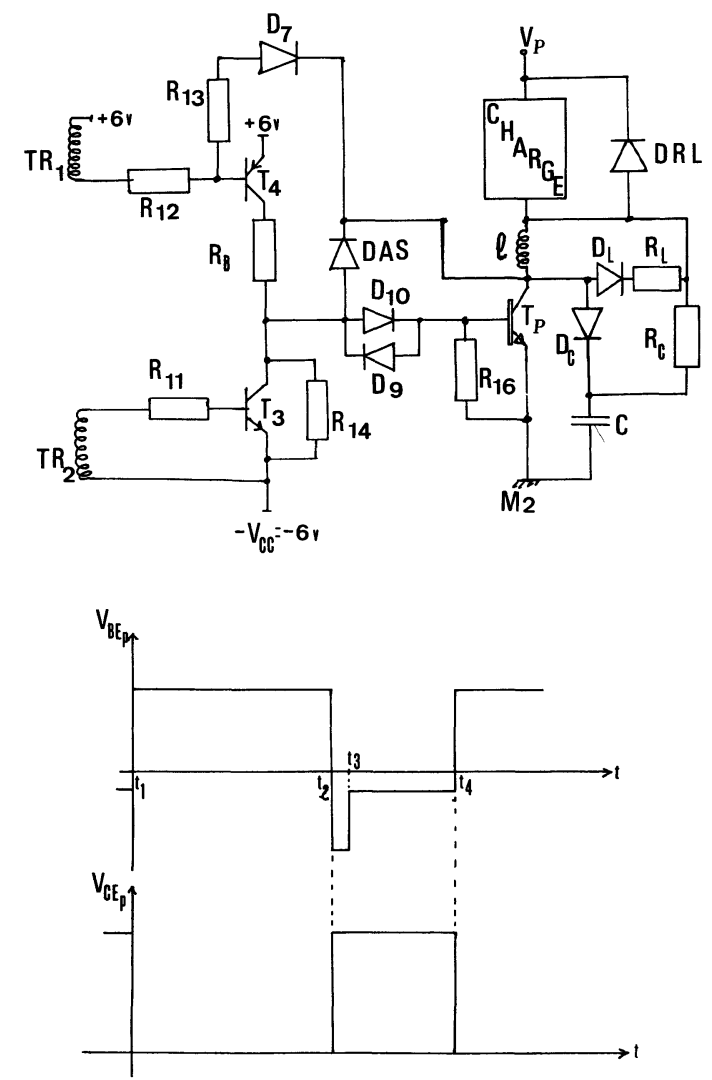

Fig. 6. - Schéma du bistable de commande et protection des transistors de puissance et oscillogramme des tensions baseémetteur $V_{\mathrm{BEP}}$ et collecteur-émetteur $V_{\mathrm{CEP}}$ correspondant au fonctionnement en commutation.

[Diagram of the control and protection bistable of the power transistors and voltage oscillogram of base-emitter $V_{\mathrm{BEP}}$ and collector-emitter $V_{\text {CEP }}$ corresponding to operation at commutation.] 
L'impulsion de commande de saturation est transmise par l'intermédiaire du transformateur d'impulsion TRI et $R_{12}$ à la base du transistor $T_{4}$ qui se sature, ce qui permet d'alimenter la base du transistor de puissance par un courant :

$$
I_{\mathrm{B}}=\frac{V_{\mathrm{CC}}-\left(V_{\mathrm{CET}_{4}}+V_{\mathrm{BET}_{\mathrm{p}}}\right)}{\mathrm{R}_{\mathrm{B}}} .
$$

On peut donc régler la saturation de $T_{p}$ par action sur $\mathrm{R}_{\mathrm{B}}$. La tension $V_{\mathrm{CET}_{\mathrm{p}}}$ s'effondre, ce qui met la cathode de la diode $\mathrm{D}_{7}$ à la masse, à la tension de saturation du transistor $T_{p}$. Le pont constitué par $R_{12}$, $R_{13}$ et la diode $D_{7}$ conductrice, maintient la saturation de $\mathrm{T}_{4}$ et par conséquent le courant $I_{\mathrm{B}}$. C'est la phase de mémorisation du signal de commande qui correspond à l'intervalle de temps $\left(t_{1}, t_{2}\right)$. Toutefois, le taux de saturation de $T_{p}$ dépend du courant $I_{B}$ fourni à sa base et du courant $I_{C}$ de collecteur. La diode d'antisaturation $D_{A S}$ évite une saturation excessive du transistor qui augmenterait le temps de stockage.

La diode $\mathrm{D}_{\mathrm{AS}}$ et la diode $\mathrm{D}_{10}$ fixent la tension du transistor de puissance à $V_{\mathrm{CBT}_{\mathrm{p}}}=0$. La tension collecteur émetteur du transistor de puissance ne peut descendre en dessous de la tension base émetteur et le transistor fonctionne en régime de quasisaturation.

3.2 Commande du blocage. - L'impulsion de commande du blocage est transmise pendant l'intervalle de temps $\left(t_{2}, t_{3}\right)$ par l'intermédiaire du transformateur TR 2 et $R_{11}$ à la base du transistor $T_{3}$ qui se sature. La tension négative appliquée à la base du transistor de puissance entraîne son blocage donc celui de $D_{7}$, ce qui annule la phase de mémorisation décrite ci-dessus.

A l'instant $t_{3}$ l'impulsion de commande de blocage disparaît et $T_{3}$ se bloque. La base du transistor de puissance $T_{p}$ est polarisée par une fraction de tension négative par l'intermédiaire de la résistance $R_{14}$, de la diode $D_{9}$ et de la résistance $R_{16}$.

$$
V_{\mathrm{BEP}}=\frac{-\mathrm{R}_{16} V_{\mathrm{CC}}}{\mathrm{R}_{14}+\mathrm{R}_{16}}
$$

et ceci jusqu'à l'instant $t_{4}$ d'apparition d'une nouvelle impulsion de commande de saturation.

3.3 Protection ACtive CONTRE LeS SURINTENSITÉS. - En cas de surintensité, le transistor se désature car le courant $I_{\mathrm{B}}$ est limité par la résistance $\mathrm{R}_{\mathrm{B}}$. La tension $V_{\mathrm{CE}}$ augmente entraînant le blocage de la diode $\mathrm{D}_{7}$ donc le blocage du transistor $\mathrm{T}_{4}$. La base du transistor $\mathrm{T}_{\mathrm{p}}$ est alors soumise à une tension négative qui le bloque comme dans la phase de mémorisation du blocage décrite ci-dessus.

Le transistor de puissance ne conduit à nouveau que par envoi d'une impulsion de commande de saturation. Il est à noter que le choix du courant $I_{\mathrm{C} \text { MAX }}$ est obtenu par action sur la résistance $R_{B}$ dans les limites du courant maximum $I_{\mathrm{CM}}$ admissible par le transistor. Le bistable qui a été réalisé assure non seulement la saturation et le blocage corrects du transistor de puissance, mais aussi la protection de ce dernier contre les surintensités. Cette protection active contre les surintensités protège à la fois la charge et le transistor et augmente la fiabilité du montage.

3.4 ETUde du RÉSEAU D'AIDE a LA COMMUTATION. Le réseau d'aide à la commutation à la fermeture (Fig. 6) est constitué par une inductance $l$, une diode $D_{L}$ et une résistance $R_{L}$. Au moment de la fermeture, l'inductance retarde la montée du courant dans le transistor, ce qui améliore le cycle de commutation et diminue la puissance dissipée dans le transistor puisqu'il n'y a plus présence simultanée de tension $V_{\text {CEP }}$ et de courant $I_{\text {CP. }}$.

L'énergie stockée dans l'inductance $l$ est ensuite restituée dans la résistance $R_{L}$.

Le réseau d'aide à la commutation à l'ouverture est constitué par une capacité $C$, une diode $D_{c}$ et une résistance $R_{C}$. La capacité $C$ retarde la montée en tension et évite la présence simultanée de la tension et du courant au moment de la commutation, ce qui diminue les contraintes et les pertes en commutation au niveau du transistor. L'énergie stockée dans la capacité est dissipée dans la résistance $\mathbf{R}_{C}$.

Ainsi le choix convenable d'un réseau d'aide à la commutation entraîne une diminution des pertes totales [5]. Enfin la dissipation de l'énergie s'effectuant dans les résistances, le transistor de puissance ne subit pas de contraintes thermiques.

4. Onduleur triphase. - L'ensemble générateur photovoltaïque-adaptateur que nous venons de décrire constitue une source d'énergie continue à puissance variable avec l'ensoleillement. Pour alimenter le moteur asynchrone de la pompe immergée à partir de cette énergie, nous avons intercalé un onduleur à transistor [6]. Le montage global (Fig. 7) comprend un générateur photovoltaïque avec sa capacité de

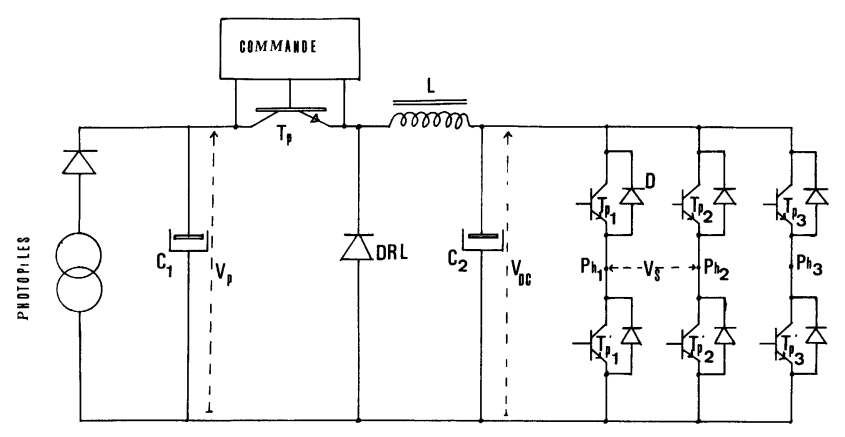

Fig. 7. - Schéma de principe de l'ensemble hacheur adaptateuronduleur triphasé à fréquence variable.

[Skeleton diagram of the three-phase variable power chopper adaptor inverter set.] 
filtrage $\mathrm{C}_{1}$, le hacheur adaptateur constitué par le transistor $T_{p}$, la diode de roue libre $D_{R L}$ et l'inductance de filtrage $\mathrm{L}$, et un onduleur triphasé avec une capacité de filtrage d'entrée $\mathrm{C}_{2}$. La commande de cet onduleur triphasé (Fig. 8) est constituée par un générateur de signaux triphasés, des circuits déphaseurs et de mise en forme, des circuits d'élaboration des impulsions, et les bistables de commande des transistors de puissance.

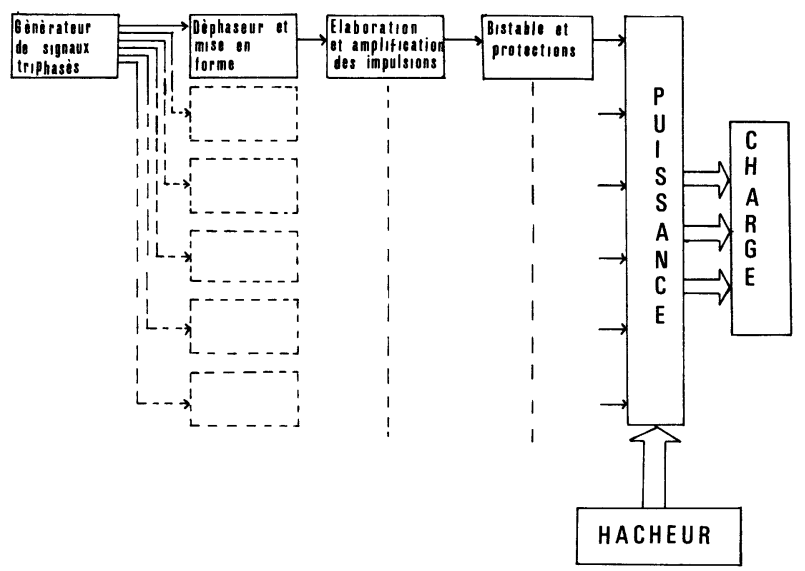

Fig. 8. - Schéma synoptique de l'onduleur.

[Synoptic diagram of the inverter.]

Nous avons préféré l'utilisation de transistor à celle de thyristors pour des questions de rendement.

Pour fonctionner à couple maximum, le moteur asynchrone doit fonctionner à $V_{\mathrm{s}} / f_{\mathrm{s}}=$ Cte avec $V_{\mathrm{S}}$ tension efficace appliquée au stator et $f_{\mathrm{S}}$ fréquence de cette onde de tension. La commande des transistors de puissance d'une même branche étant complémentaire, nous obtenons une tension composée égale à la tension continue d'entrée de l'onduleur $V_{\mathrm{DC}}$ pendant $120^{\circ}$.

La tension efficace d'alimentation du stator du moteur asynchrone est proportionnelle à la tension continue d'entrée $V_{\mathrm{DC}}$.

$$
V_{\mathrm{S}}=\sqrt{\frac{2}{3}} V_{\mathrm{DC}}
$$

Le fonctionnement à couple maximum est obtenu en pilotant un convertisseur tension fréquence par la

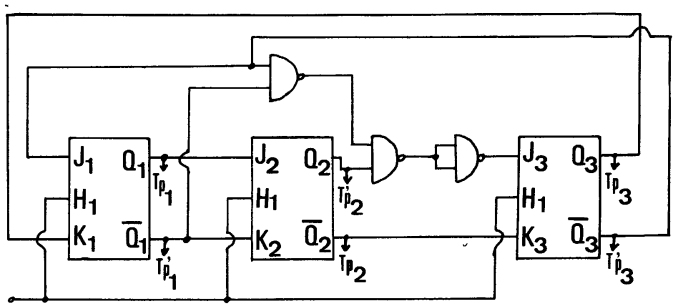

Fig. 9. - Compteur en anneau qui élabore les impulsions de commande triphasée.

[Loop counter which produces the three-phase control pulses.] tension d'entrée continue $V_{\mathrm{DC}}$. Un compteur en anneau (Fig. 9) permet d'obtenir les 6 signaux de commande des transistors de puissance. Afin d'éviter une conduction simultanée d'une même branche de l'onduleur, ces signaux sont retardés puis remis en forme avant d'être appliqués à un bistable de commande identique à celui du hacheur.

Le prototype a été construit avec des transistors MOTOROLA MJ $10015\left(V_{\mathrm{CEO}}=400 \mathrm{~V} I_{\mathrm{CM}}=50 \mathrm{~A}\right)$ mais on peut utiliser des transistors SESCOSEM ESM 870.

Le choix d'une technologie à transistor de puissance permet d'utiliser une fréquence élevée pour le hacheur de tête $(\simeq 2 \mathrm{kHz})$, ce qui conduit à une réduction des dimensions des cellules de filtrage.

Le fonctionnement de l'onduleur triphasé est caractérisé par les oscillogrammes des figures 10 et 11 qui représentent :

- la tension entre phase,

- le courant dans une phase,

- la tension $V_{\text {BET }_{\mathbf{p}}}$ d'un transistor de puissance,

- la tension $V_{\text {CET }_{\mathbf{p}}}$ de ce transistor de puissance.

Ces oscillogrammes montrent que la machine asynchrone est le siège de pertes supplémentaires dues à la forme du courant qui l'alimente. Pour des puissances supérieures à $10 \mathrm{~kW}$, il serait souhaitable d'effectuer une alimentation en courant du moteur en lui imposant une valeur sinusoïdale.

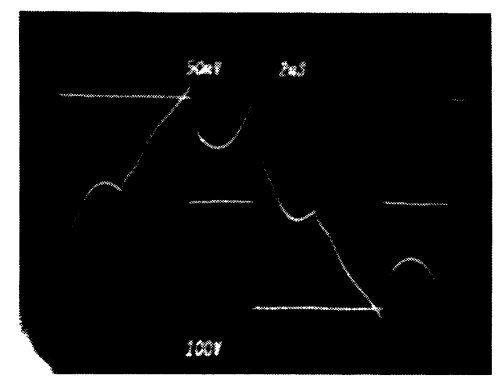

TENSION COMPOSEE

COUR A N T

DANS UNE

PHASE

$(100 \mathrm{mv} \rightarrow 10 \mathrm{~A})$

Fig. 10. - Oscillogramme des courant et tension entre deux phases pour une puissance absorbée de $2 \mathrm{~kW}, 220 \mathrm{~V}, 50 \mathrm{~Hz}$.

[Oscillogram of phase-to-phase current and voltage for an input power of $2 \mathrm{~kW}, 220 \mathrm{~V}, 50 \mathrm{~Hz}$.]

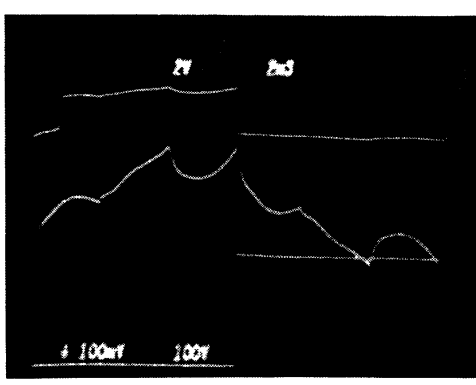

$V_{B E}$

COURANT DANS

UNE PHASE

$(100 \mathrm{mv} \rightarrow 10 \mathrm{~A})$

$V_{\text {CE }}$

Fig. 11. - Oscillogramme des tensions $V_{\mathrm{BE}}$ et $V_{\mathrm{CE}}$ pour une puissance absorbée à $2 \mathrm{~kW}, 220 \mathrm{~V}, 50 \mathrm{~Hz}$.

[Oscillogram of voltages $V_{\mathrm{BE}}$ and $V_{\mathrm{CE}}$ for input power of $2 \mathrm{~kW}$, $220 \mathrm{~V}, 50 \mathrm{~Hz}$.] 
La sécurité de fonctionnement de l'ensemble est excellente grâce aux circuits d'aide à la commutation associés à chaque transistor de puissance et aux protections actives contre les courts-circuits qui protègent aussi bien l'électronique que le groupe motopompe.

5. Résultats expérimentaux. - Les essais ont été effectués avec un groupe électropompe immergé Guinard de type SP 611 T 3, 2,2 kW, $220 \mathrm{~V}, 50 \mathrm{~Hz}$. Les variations de la puissance électrique continue fournie à l'onduleur ont été obtenues en maintenant la tension d'entrée du hacheur constante durant toutes les mesures et en faisant varier le rapport cyclique. La différence de niveau entre la bouche de sortie de l'eau et la surface de l'eau au fond du puits est de $9 \mathrm{~m}$. Dans ces conditions, nous avons tracé (Fig. 12) en fonction de la puissance $P_{\mathrm{a}}$ absorbée par le hacheur de tête les variations du débit $D$, du rendement hydraulique pompe centrifuge-moteur asynchrone $\eta_{H}$, du rendement de l'ensemble électronique hacheuronduleur $\eta_{\mathrm{E}}$ et du rendement total de l'installation de pompage $\eta_{\mathrm{T}}$.

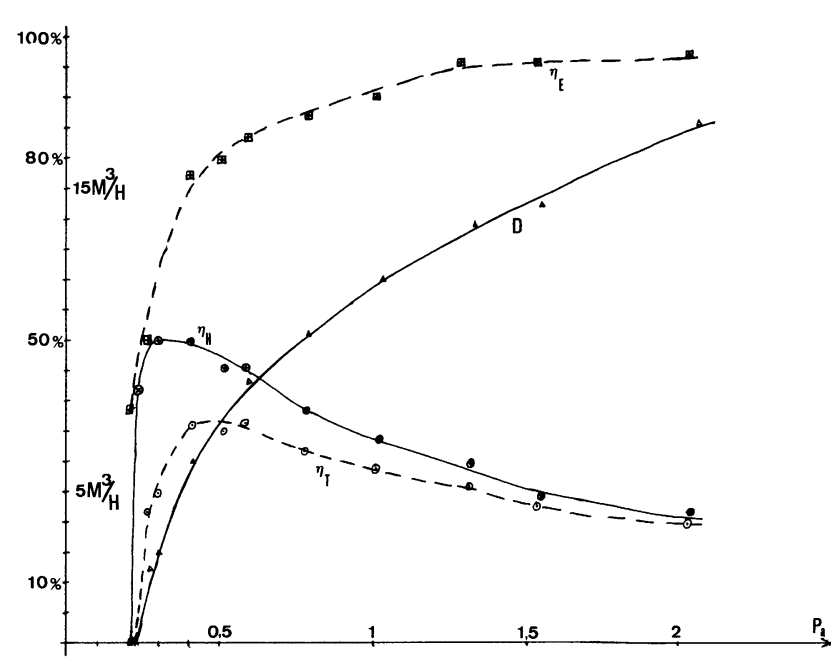

Fig. 12. - Variations du débit $D\left(\mathrm{~m}^{3}\right)$, du rendement hydraulique $\eta_{\mathrm{H}}$, du rendement de l'électronique $\eta_{\mathrm{E}}$, et du rendement total de l'installation de pompage en fonction de la puissance absorbée $P_{\mathrm{a}}$.

[Variations in flow $\left(\mathrm{m}^{3}\right)$, water output $\eta_{\mathrm{H}}$, electronic efficiency $\eta_{\mathrm{E}}$, total output of the pumping installation on the basis of input power $P_{\text {a. }}$.]

Les mesures ont été effectuées sur toute la gamme de puissance qui s'étend jusqu'à la puissance nominale $\mathrm{du}$ moteur asynchrone, soit $2,2 \mathrm{~kW}$ correspondant à une tension alternative de $220 \mathrm{~V}$ et de fréquence $50 \mathrm{~Hz}$. On peut constater sur cette courbe (Fig. 12) que le moteur qui alimente la pompe centrifuge doit absorber une puissance minimum de $250 \mathrm{~W}$ pour que l'exhaure de l'eau soit possible. On peut aussi remarquer que le rendement de l'électronique est excellent $\left(\eta_{\text {MAX }} \simeq 95 \%\right)$ alors que le rendement hydraulique varie de $50 \%$ à $25 \%$.

Ce groupe de pompage sera alimenté par un générateur photovoltaïque de puissance crête $1,5 \mathrm{~kW}$ pour un ensoleillement de $1 \mathrm{~kW} / \mathrm{m}^{2}$. La courbe qui représente les variations de la puissance absorbée $P_{\mathrm{a}}$ (Fig. 13) en fonction de la fréquence de fonctionnement de l'onduleur indique qu'il doit fonctionner à $44 \mathrm{~Hz}$ pour la puissance crête. On peut alors en déduire la tension efficace maximale à la sortie de l'onduleur à partir de la loi tension fréquence qui permet le fonctionnement à couple maximum :

$$
\frac{V_{\text {eff }}}{55}=\frac{220}{50} V_{\text {eff }}=193,6 \mathrm{~V} \text {. }
$$

Compte tenu de la forme de la tension entre phase appliquée au moteur, la relation entre la tension efficace et la tension continue $V_{\mathrm{DC}}$ à l'entrée de l'onduleur est

$$
V_{\text {eff }}=\sqrt{\frac{2}{3}} V_{\mathrm{DC}} .
$$

Ainsi la tension minimum de fonctionnement du générateur photovoltaïque sera :

$$
V_{\mathrm{P}}=\sqrt{\frac{3}{2}} V_{\text {eff }}=237,1 \mathrm{~V}
$$

soit 10 branches parallèles de 16 panneaux BPX $47 \mathrm{~A}$ montés en série puisque pour un panneau $V_{\text {opt }} \simeq 15 \mathrm{~V}$.

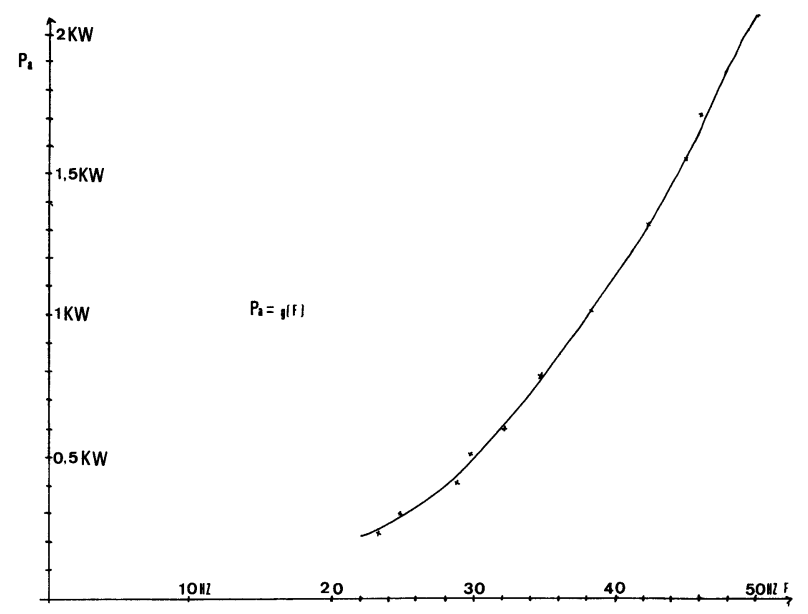

Fig. 13. - Variations de la puissance absorbée $P_{\mathrm{a}}$ en fonction de la fréquence de l'onduleur.

[Variations in input power on the basis of the inverter frequency.]

A partir de la courbe des variations $D$ du débit en fonction de la puissance et en admettant que cette puissance varie sinusoïdalement au cours d'une journée, on peut en déduire (Fig. 14) l'évolution du débit en fonction du temps pendant la période d'ensoleillement. 


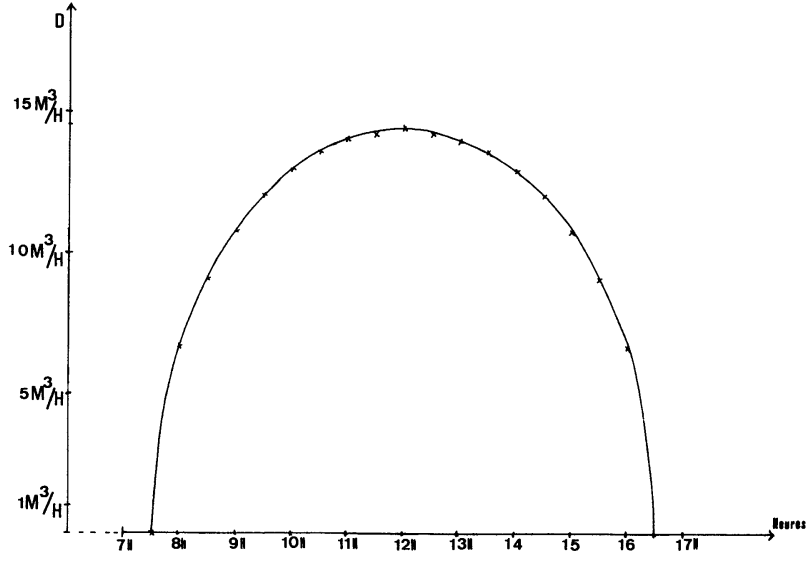

Fig. 14. - Variations du débit de la pompe au cours d'une journée très ensoleillée pour une hauteur manométrique de $9 \mathrm{~m}$

[Variations in flow of the pump in the course of a very sunny day at a pressure gauge height of nine metres.]

Le seuil de fonctionnement de $250 \mathrm{~W}$ est atteint à $8 \mathrm{~h} 30$ et $17 \mathrm{~h} 30$ par un ensoleillement de $167 \mathrm{~W} / \mathrm{m}^{2}$. Dans ces conditions, le pompage journalier est de $100 \mathrm{~m}^{3}$ à une profondeur de $9 \mathrm{~m}$.

Compte tenu de l'excellent rendement de l'électronique, ces résultats ne peuvent être sensiblement améliorés qu'en faisant des efforts sur la conception du groupe immergé qui doit par ailleurs être adapté à la profondeur du puits et au volume d'eau à pomper chaque jour.

6. Conclusion. - Nous nous sommes efforcés au cours de cette étude d'optimiser une station de pompage alimentée par un générateur photovoltaïque. Dans un premier temps, nous avons imposé au générateur photovoltaïque un fonctionnement à puissance maximale quel que soit l'ensoleillement au moyen d'un convertisseur d'énergie continue-continue réalisé à l'aide d'un hacheur dévolteur asservi. Ce convertisseur statique peut adapter n'importe quel récepteur au générateur photovoltaïque et l'ensemble photopilehacheur constitue un générateur photovoltaïque idéal qui fournit avec un excellent rendement (97\%) la puissance maximale disponible aux bornes des photopiles.

Avec ce dispositif, il est possible d'alimenter un groupe de pompage conventionnel constitué par un moteur continu en surface qui entraîne soit une tringlerie reliée à une pompe à piston située au fond du puits, soit un axe vertical couplé à une pompe centrifuge immergée, soit une hydropompe du type Vergnet. Nous avons préféré utiliser un groupe immergé constitué par une pompe centrifuge accouplée au fond du puits à un moteur asynchrone alimenté par un câble électrique.

Nous remplaçons ainsi une transmission mécanique coûteuse et source de pertes supplémentaires par une transmission électrique à très faibles pertes particulièrement bien adaptée à un puits ou forage profond.

En outre le moteur asynchrone peu coûteux et d'entretien quasiment nul associé à la pompe centrifuge constitue un groupe immergé de grande diffusion.

Le développement industriel d'onduleurs triphasés de grande fiabilité et de coût moins élevé devrait permettre une généralisation de ce type de station de pompage.

Enfin, compte tenu des limites technologiques actuelles des transistors de puissance, on peut extrapoler les résultats obtenus avec une puissance de $2 \mathrm{~kW}$ à des installations de puissance crête atteignant la cinquantaine de kilowatts.

Remerciements. - Les auteurs tiennent à remercier M. Henri Foch Maître de Conférences à l'ENSEEIHT, pour sa collaboration efficace et pour les précieux échanges d'information.

\section{Bibliographie}

[1] Diouf, A. et GuEYE, P. en collaboration avec le L.E.P., Etude et réalisation d'une pompe solaire à photopiles. Mémoire de fin d'étude Ingénieur Technologue en Génie Electrique, ENSUT, Université de Dakar, Avril 1976.

[2] Barlaud, M. et Masselot, C., Optimisation d'une station de pompage alimenté par un générateur photovoltaïque. Photovoltaic Solar Energy Conference Luxembourg 27 30 sept. 1977.

[3] Barlaud, M. et Masselot, C., Séminaire d'Energie Solaire. Centre International de Physique Théorique de Trieste, sept. 1978
[4] Pickrell, R. L., O'Sullivan, G. and Merril, W. C., An Inverter/Controller subsystem optimized for photovoltaic applications, Thirteenth IEEE Photovoltaic Specialists Conference, 1978

[5] Peter, J. M., Les réseaux d'aide à la commutation. Le transistor de puissance dans son environnement SESCOSEM 78.

[6] Foch, Trannoy, Roux, Utilisation rationnelle des transistors de puissance haute tension pour l'alimentation d'une machine asynchrone à partir du réseau $380 \mathrm{~V}, 50 \mathrm{~Hz}$. Journée Internationale d'étude sur l'utilisation des semi-conducteurs AIM Liège (13-14 nov. 78). 